\title{
Paternal 20q13.2q13.3 microdeletion syndrome
}

INSERM

\section{Source}

INSERM. (1999). Orphanet: an online rare disease and orphan drug data base. Paternal

20q13.2q13.3 microdeletion syndrome. ORPHA:261304

Paternal 20q13.2q13.3 microdeletion syndrome is a recently described syndrome

characterized by severe pre- and post-natal growth retardation, microcephaly, intractable feeding difficulties, mild psychomotor retardation, hypotonia and facial dysmorphism. 